\title{
Lesion-based radiotherapy of the ears, lips and eyelids for skin cancer
}

\section{Abstract}

Purpose: This study is a retrospective audit of radiotherapy (RT) for skin cancer of the ear, eyelid, and lip in Sydney, Australia. The growth of referrals to a tertiary radiation oncology service over a specific time period were also assessed.

Materials and methods: The records of patients who received RT to the external ear, eyelid or lip between January 12007 and April 302020 were reviewed. Patient, tumour, treatment and outcome factors were recorded.

Results: 147 patients with a mean age of 73 years (range: 33-96) were identified as eligible for inclusion. 165 lesions were treated and 18 patients had multiple treatment events. Of all the treated lesions, 81 were basal cell carcinoma $(49.2 \%), 65$ cutaneous squamous cell carcinoma (39.4\%), 7 lentigo maligna (LM) (4.2\%), 2 sebaceous carcinoma (1.2\%), 2 merkel cell carcinoma (1.2\%), 4 Bowen's disease (2.4\%), 2 actinic change $(1.2 \%)$ and 2 keloid treatments $(1.2 \%)$. The mean follow-up was 42 weeks. Definitive RT, that is, RT given as primary treatment rather than post operatively, was given in 108 cases. There were five cases of BCC recurrence and three cases of $\mathrm{cSCC}$ recurrence within 3 - 61 months. These were successfully salvaged in six cases $(75 \%)$. Late side effects were seen in 26 patients with abnormal cosmetic results being the most common late effect $(6 / 26 ; 23.1 \%)$.

Conclusion: Lesion-based RT for skin cancer of the ear, eyelid, and lip can be delivered safely and is effective. Definitive RT may be preferable to surgery especially when tissue loss may lead to poor functional and cosmetic outcomes.

Keywords: radiotherapy, pinna, ear, eyelid, lip, skin cancer, Australia
Volume 8 Issue I - 202I

Anthony Tanous,' David Tighe,' Julie Bartley, ${ }^{2}$ Gavin Gottschalk, ${ }^{3}$ Tanya Gilmour, ${ }^{4}$ Nicholas Lotz, ${ }^{5}$ Gerald B. Fogarty ${ }^{2}$

'Faculty of Medicine, University of New South Wales, Australia ${ }^{2}$ Genesis Care, Mater Hospital, Australia

${ }^{3}$ Chatswood Dermatology Centre, Australia

${ }^{4}$ North Shore Dermatology \& Specialist Skin Cancer Centre, Australia

${ }^{5}$ North Shore Cosmetic Surgery, Level I,Australia

Correspondence: Prof Gerald Fogarty, Radiation Oncology, Mater Sydney, Crows Nest, NSW Australia, $\mathrm{Ph}+6129458$ 8050, Fax +6I 299292687 , Email Gerald.Fogarty@genesiscare.com

Received: March 24, 2021 | Published: April 07, 2021

\section{Introduction}

Skin cancer is common in Australia and is increasing in incidence. Amongst skin malignancies, keratinocytic cancer is the most common. ${ }^{1}$ Keratinocytic cancers include basal cell carcinoma (BCC) and cutaneous squamous cell carcinoma (cSCC). Surgery is often used but always involves tissue sacrifice, which may impact functional and cosmetic outcomes, especially in the head and neck region (Figure 1). ${ }^{2}$ Radiotherapy (RT) can be given in the post-operative scenario (PORT) when high risk features, such as positive margins or perineural invasion (PNI), are seen, or as definitive treatment when RT is given as the sole treatment. Definitive RT offers comparable cure rates, ${ }^{3}$ conserves tissue, and may provide better functional and cosmetic outcomes in select sites.

Figure I Three cases following surgery of the external ear for skin cancers. Tissue sacrifice associated with surgery has led to inferior cosmetic outcomes.

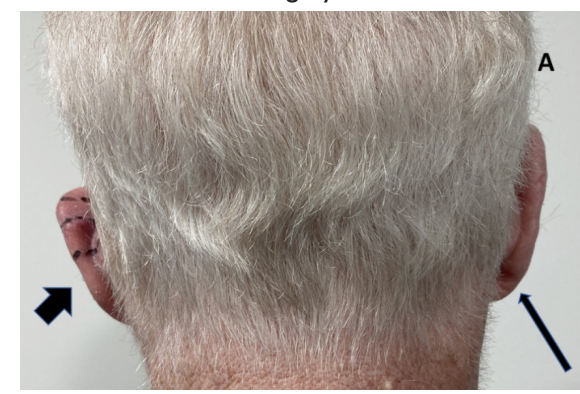

Case A. Photo is taken from behind to compare the operated left ear with the unoperated right ear.The left ear (broad arrow) had had a lesion removed from the mid pinna with primary closure and needed post opertaive radiotherapy (PORT) or adjuvant RT due to a positive margin. Note the smaller size of the operated pinna (broad arrow) versus the right unoperated pinna (thin arrow).

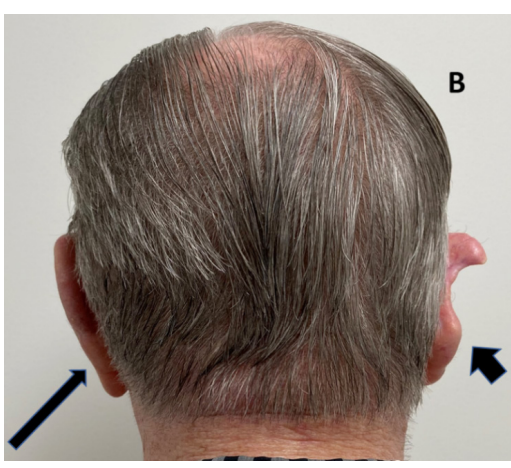

Case B. Photo is taken from behind to compare the operated right ear (broad arrow) with the unoperated left ear (thin arrow). The right ear had had a lesion removed from the mid pinna with primary closure resulting in significant deformity of the pinna.

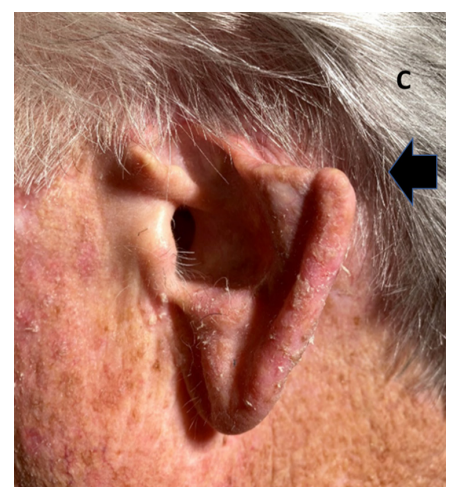

Case $C$. This left ear had had a lesion removed from the upper and middle pinna with amputation of the top part of the pinna (broad arrow). 
Difficult anatomical sites for surgery include the external ear, eyelid and lip. These organs are important for adequate function as well as for cosmesis. External ears help to concentrate sound but are also needed as support for earrings, glasses, hearing aids and, in these COVID times, face masks. Definitive RT enables tissue conservation in these sites. This is a retrospective audit of one radiation oncologist's (RO) work (GBF) and aims to provide modern Australian retrospective data on lesion-based definitive RT of the ears, lips and eyelids.

\section{Methods}

\section{Ethics approval}

St Vincent's Hospital Human Research Ethics Committee provided ethical approval on 12 December 2019 (ETH13048).

\section{Patient selection}

Patients were included in this retrospective, single clinician audit if they were aged over 18 years and had a skin cancer of the ear, eyelids and/or lips that was planned for RT treatment in Sydney between January 2007 and April 2020. Patient factors, tumour factors, treatment factors and outcomes data were collected by assessing individual medical records. The recorded patient factors included date of birth and age at simulation, gender, post-code of residency at treatment, referring practitioner and immunosuppression status. Immunosuppression status was defined as patients on long-term corticosteroids, or long-term chemotherapy such as methotrexate; chronic blood disorders such as lupus and leukaemia; those with organ transplants on immune suppressive drugs, and human immunodeficiency virus infection (HIV).

Tumour clinical factors were tumour size, depth, location, and a history of previous treatment to the lesion prior to RT. Tumour histological factors were available if there was a biopsy prior to referral which allowed the histological type to be accessed. In patients who underwent an excisional biopsy, more data was available including histological depth, differentiation, presence of adverse factors such as PNI, close or positive margins, and lymph-vascular space invasion (LVSI).

The external ear was broken into three parts: the pinna (consisting of the helix, anti-helix, lobulus, tragus, and the scapha) $)^{4}$, the conchal bowl, and the external auditory meatus (EAM) (Figure 2).

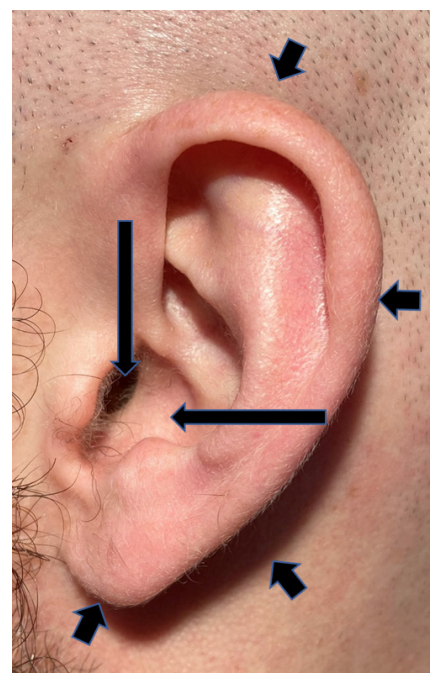

Figure 2 Normal anatomy of the ear.The pinna is defined by the short arrows, the conchal bowl by the long horizontal arrow, and the external auditory meatus by the long vertical arrow.
The lip and the eyelid were described using upper and lower, with the inner canthus included as an anatomical part of the eyelid for this study.

Treatment factors included radiotherapy modality and role, dosage, fractionation, radiotherapy start and completion dates, any unplanned breaks, and field size. The role of radiotherapy was defined as either radical or palliative; radical was further defined into definitive or adjuvant. Field size was measured either during planning by the prescribing $\mathrm{RO}$ or retrospectively by the research team using a scale and images. Outcome factors (when documented) included acute side effects based on the Common Terminology Criteria of Adverse Effects V5 (CTCAE-V5), late side effects, recurrence, salvage therapy, cosmetic outcome, and length of post-treatment follow-up. Acute side effects were defined as occurring between 0-3 months post RT, and late side effects were defined as occurring at least six months post RT. ${ }^{5}$ Local recurrence was defined as recurrence within the previous treatment field.

\section{Radiotherapy process}

Initially, each patient was assessed for RT suitability. Assessment factors included lesion history, RT history, and the availability to attend the clinic for fractionation. The RO examined the patient to determine the extent of the lesion and the areas requiring treatment. If RT was deemed suitable, informed consent was obtained and the patient proceeded to RT planning. Here, the patient was immobilised in a comfortable and reproducible position. A mask was used for linear accelerator-based treatments.

Different treatment modalities were employed and depended upon machine availability and the nature of the lesion. The most common treatment modalities were electron beam radiation (usually 6 megavolts $(\mathrm{MeV})$ and $9 \mathrm{MeV})$, megavoltage photon beam radiation (MV), and superficial $\mathrm{x}$-ray radiation therapy (SXRT) using the $100 \mathrm{kV}$ beam of a Xstrahl 300 machine (Xstrahl Ltd, Surrey, UK). The superficial machine was especially practical in older patients who could not tolerate a mask (Figure 3).

When using megavoltage electrons of these energies, tissue equivalent material called "bolus" or 'build up' is used to ensure that the right dose reaches the target, or to 'soak up' any dose that may be heading for normal tissue (Figure 4).

Figure 3 Superficial $x$-ray radiation therapy (SXRT) with a Xstrahl 300 machine was often used, especially in older patients who could not tolerate a mask. This patient had SXRT treatment to bilateral biopsy-proven BCC of the pinnas.

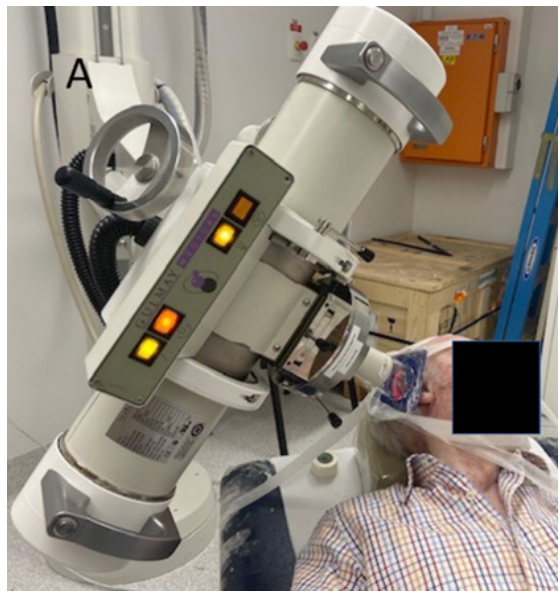

Figure 3A Treating the right pinna. 


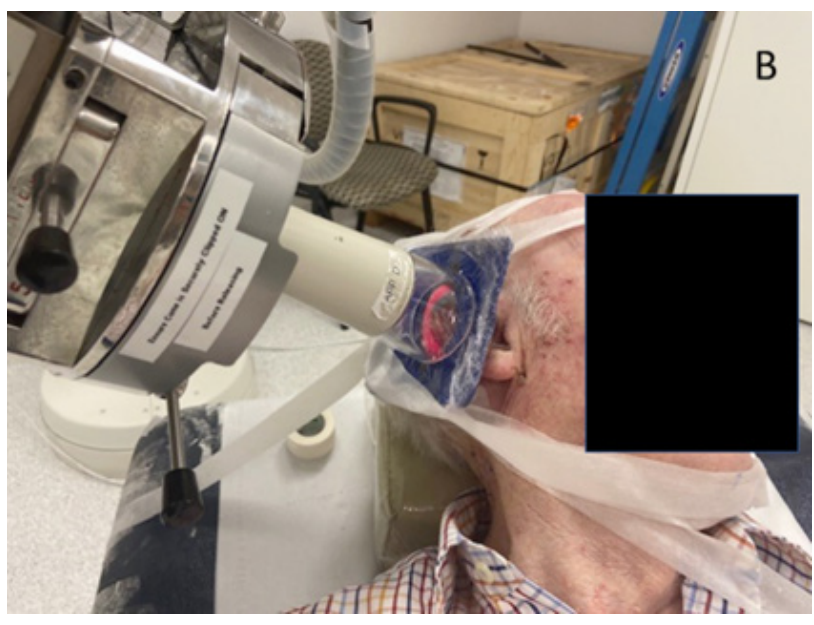

Figure 3B Treating the right pinna close up. Note the pink material (bolus) used to ensure sufficient dose to the edge of the pinna.

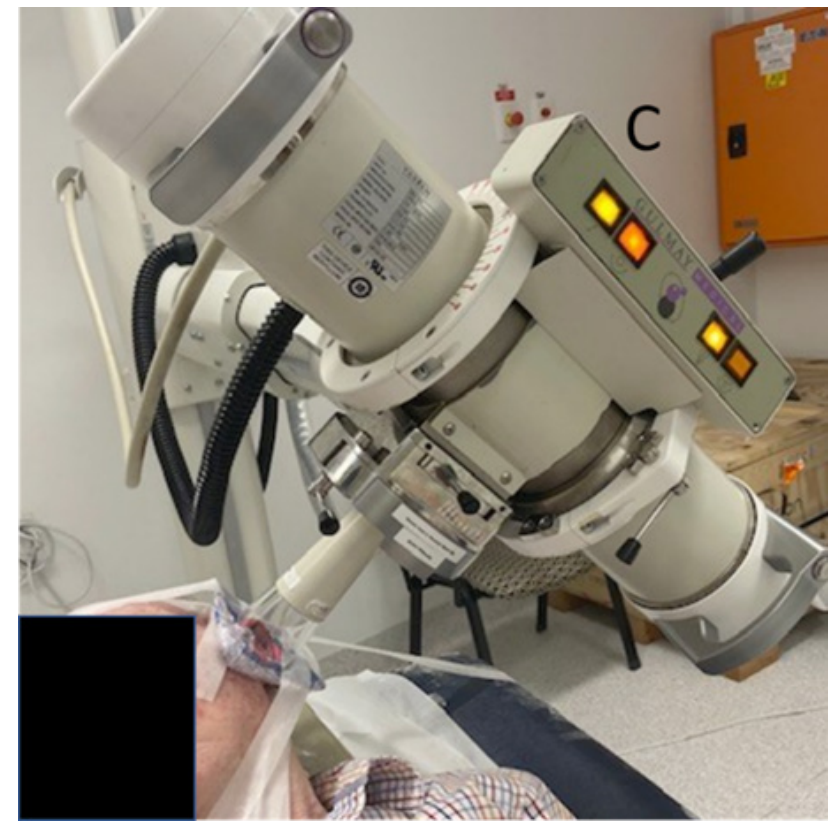

Figure 3C Treating the left pinna.

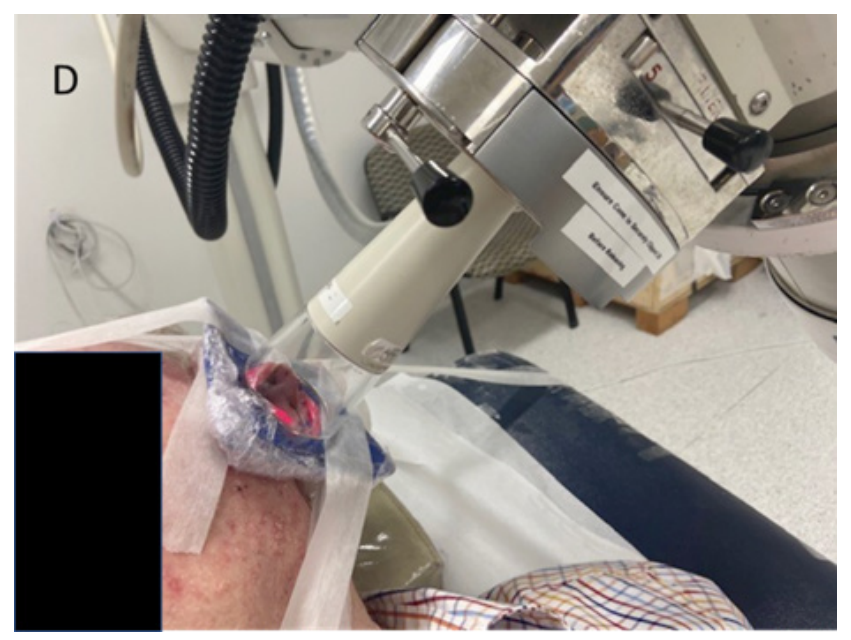

Figure 3D Treating the left pinna close up.Again, note the pink bolus material used to ensure sufficient dose to the edge of the pinna.
Figure 4 Use of bolus for megavoltage electrons to achieve adequate dose to the lesion.

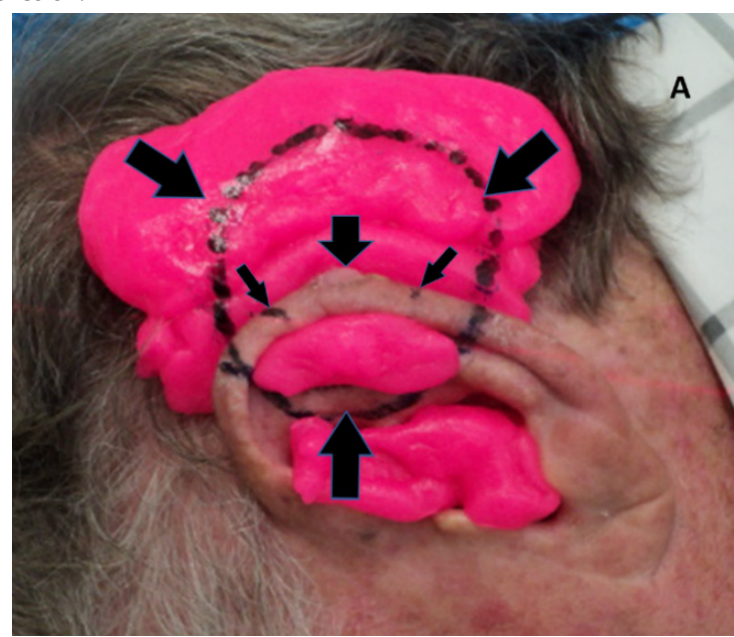

Figure 4A Treatment set up for an electron plan for a lesion on the edge of the mid-pinna. The short thick vertical arrow pointing down shows an exophytic lesion. The short thin diagonal arrows show the extent of the treatment field. The diagonal thick arrows and the arrow pointing up show the extent of the electric field. The pink material is bolus and it surrounds the lesion in order to build up the dose of radiation sufficient for cure. The bolus also absorbs the beam and thus protects underlying hair-bearing skin.

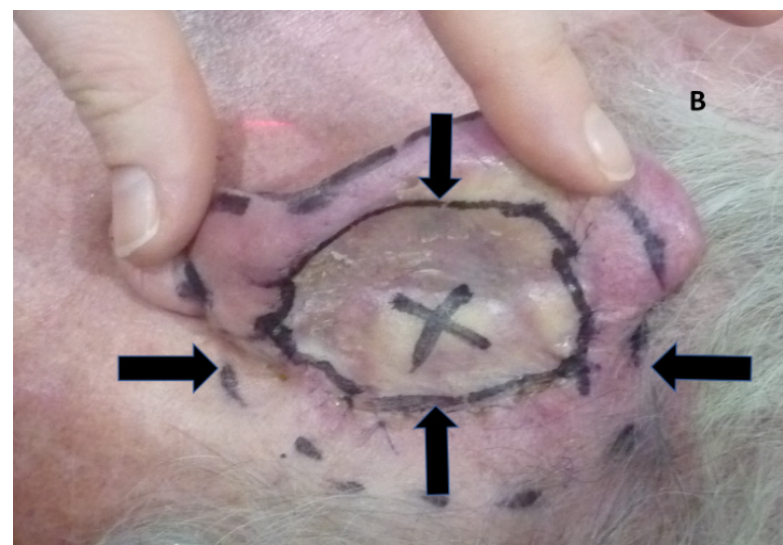

Figure 4B Using the ear flesh as bolus. The pinna has been pushed forward to expose the excision and graft on the posterior pinna. An ' $X$ ' has been placed by the surgeon to mark the location of a positive margin. Vertical arrows show the extent of the graft. Horizontal arrows show the radiation field edge.

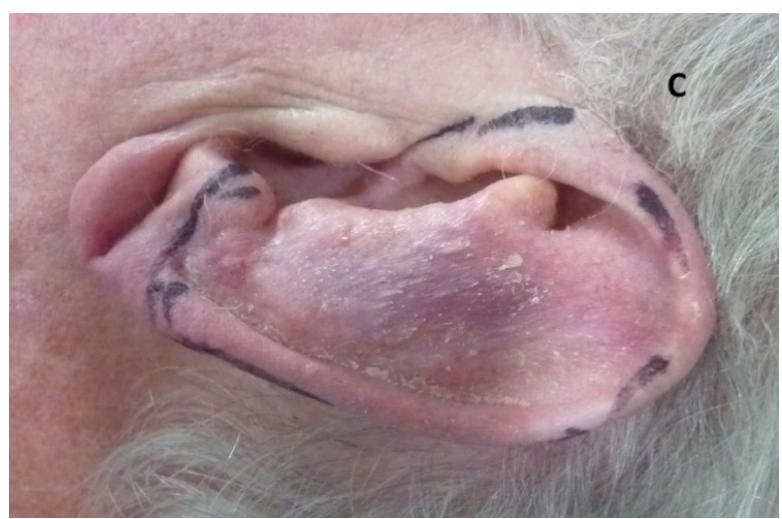

Figure 4C The pinna in $4 B$ is put into its resting position and radiation field marks are transposed from the medial side of the pinna to the lateral or outer surface of the pinna.

Citation: Tanous A, Tighe D, Bartley J, et al. Lesion-based radiotherapy of the ears, lips and eyelids for skin cancer. Int J Radiol Radiat Ther. 202I;8(I):32-42. 


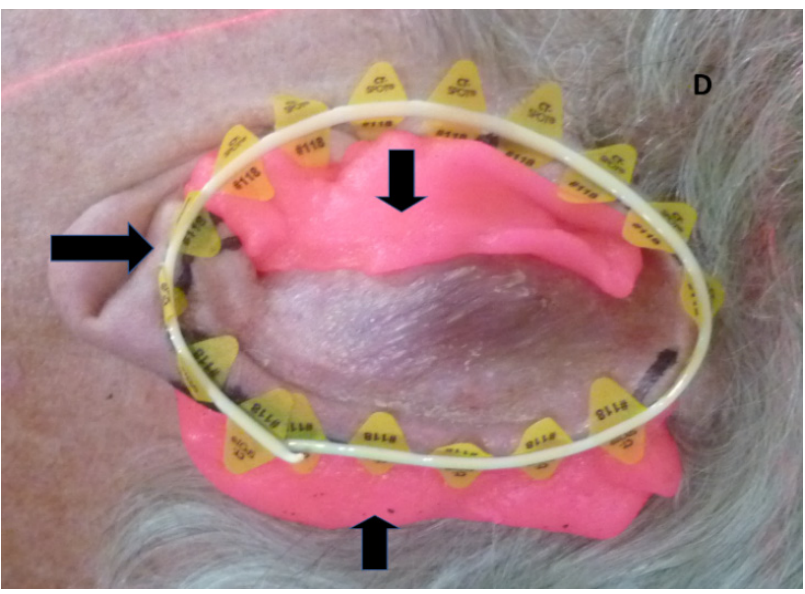

Figure 4DThe horizontal arrow shows the wire positioned over the radiation field marks to ensure the marks are captured on the computer tomography (CT) planning scan to assist contouring. The vertical arrows show the bolus in place to ensure dose build up. The anterior bolus also prevents any electron scatter into the external auditory meatus, thus decreasing side effects.

Hypofractionation, using fraction sizes of over 2 Gray (Gy), was commonly used to assist compliance. Regular RO on-treatment reviews enabled real time RT adjustment ${ }^{6}$ depending on progressive tumour and normal tissue changes with treatment. Post-treatment follow-up was carried out at 4 - 6 weeks following RT by the RO. Long term follow-up was shared with the referring doctors and often carried out solely by the referring doctor in PORT cases.

\section{Results}

\section{Patient factors}

One hundred and forty-seven patients were treated from 1 January 2007 to 30 April 2020. One hundred and fourteen were males and 33 were females. The mean age of treatment was 73 years (range: 33 to 96 years). The average follow-up length post- treatment was 42 weeks (Table 1). There were 14 immunocompromised patients (Table 2).

Table I Patient factors $(n=147)$

\begin{tabular}{lll}
\hline Patient Characteristics & Number & Percentage (\%) \\
\hline Sex & & \\
Male & 114 & 77.5 \\
Female & 33 & 22.5 \\
Age at Treatment & & \\
Mean & 73 & \\
Range & $33-96$ & \\
Follow-Up & & \\
Average Follow Up (Weeks) & 42 weeks & $4-272$ weeks \\
Immune status & & 9.5 \\
Immunocompetent & 133 & \\
Immunosuppressed & &
\end{tabular}

Table 2 Immune Status $(n=147)$

\begin{tabular}{lll}
\hline \multicolumn{2}{l}{ Mode of Immunosuppression } & \\
\hline & Frequency & Percent \\
\hline Nil immunosuppression & 133 & 90.4 \\
Corticosteroids & $\mathrm{I}$ & 0.7 \\
Lupus & $\mathrm{I}$ & 0.7 \\
HIV & 2 & 1.4 \\
Transplant & 4 & 2.7 \\
Leukaemia & 3 & 2.0 \\
Methotrexate & $\mathrm{I}$ & 0.7 \\
Chemotherapy & $\mathrm{I}$ & 0.7 \\
Lymphoma & $\mathrm{I}$ & 0.7 \\
Total & 147 & 100 \\
\hline
\end{tabular}

\section{Tumour factors}

One hundred and sixty-five lesions were treated, and 18 patients had multiple treatment events (Table 3). All depths measured were determined histopathologically except for one that was greater than 12 millimetres $(\mathrm{mm})$ and clinically described by the RO. Lesion depth was not recorded for 47 patients. The most common lesion site was on the ear, specifically the pinna. Eighteen patients had multiple lesions treated separately (147 patients, 165 lesions), and 14 treatment events spanned multiple sites (179 sites treated for 165 lesions). For example, one lesion spanned two sites of the ear, i.e. the pinna plus the EAM. The average lesion area was 3.2 centimetres $(\mathrm{cm})$ and the median lesion depth was $2.8 \mathrm{~mm}$.

Table 3 Sites Treated $(n=179$ for 165 lesions)

\begin{tabular}{lll}
\hline Tumour Characteristics & & \\
\hline Site Treated & Right & Left \\
\hline Pinna & 53 & 31 \\
Conchal Bowl & 9 & 7 \\
External Auditory Meatus & 4 & 4 \\
Upper Lip & 16 & 12 \\
Lower Lip & 8 & 10 \\
Upper Eyelid & 2 & 1 \\
Lower Eyelid & 5 & 3 \\
Inner Canthus & 4 & 10 \\
Total (by side) & 101 & 78 \\
Total = I79 sites treated & & \\
\hline
\end{tabular}

\section{Treatment factors}

Dosages were typically prescribed according to a four-week fractionation pattern measured between 45 and 65 Gy. Treatments were mostly prescribed using $6 \mathrm{MeV}$. SXRT was confined to one treatment centre (Table 4). 
Table 4 Treatment Characteristics $(n=165)$

\begin{tabular}{lll}
\hline Treatment Intent & & \\
\hline & Number & Percentage \\
\hline Definitive & 108 & 65.5 \\
Adjuvant/Post-operative & 53 & 32.1 \\
Palliative & 4 & 2.4 \\
Total & 165 & 100 \\
\hline
\end{tabular}

\section{Ears Treatment Characteristics}

\begin{tabular}{lll}
\hline \multicolumn{1}{l}{ Number } & Percentage \\
\hline Dosage & 16 & 17.4 \\
40 to 44.99 & 2 & 2.2 \\
$45-49.99$ & 20 & 21.8 \\
$50-54.99$ & 22 & 24 \\
$55-59.99$ & 13 & 14 \\
$60-64.99$ & 17 & 18.6 \\
$>65$ & 2 & 2 \\
Modality & & 16.3 \\
SXRT & 15 & 73.9 \\
6 MeV & 68 & 9.8 \\
6 MeV Photons/VMAT & 9 & 0 \\
Brachytherapy & 0 & 100 \\
Total & 92 & \\
\hline
\end{tabular}

Lips Treatment Characteristics

Number Percentage

\section{Dosage (Gy)}

\begin{tabular}{lll}
$<40$ & 4 & II.I \\
40 to 44.99 & 1 & 2.8 \\
$45-49.99$ & 3 & 8.4 \\
$50-54.99$ & 12 & 33.3 \\
$55-59.99$ & 8 & 22.2 \\
$60-64.99$ & 5 & 13.9 \\
$>65$ & 3 & 8.3 \\
MXRT & 36 & \\
6MeV & 5 & 13.9 \\
6MeV Photons/VMAT & 3 & 72.2 \\
Brachytherapy & 2 & 8.3 \\
Total & 36 & 5.6 \\
\hline
\end{tabular}

\begin{tabular}{|c|c|c|}
\hline \multicolumn{3}{|c|}{ Eyelid Treatment Characteristics } \\
\hline & Number & Percentage \\
\hline \multicolumn{3}{|l|}{ Dosage (Gy) } \\
\hline$<40$ & 3 & 15.7 \\
\hline 40 to 44.99 & 0 & 0 \\
\hline $45-49.99$ & 2 & 10.5 \\
\hline $50-54.99$ & 7 & 37 \\
\hline $55-59.99$ & I & 5.3 \\
\hline $60-64.99$ & 2 & 10.5 \\
\hline \multirow[t]{2}{*}{$>65$} & 4 & 21 \\
\hline & 19 & \\
\hline \multicolumn{3}{|l|}{ Modality } \\
\hline SXRT & 4 & 21 \\
\hline $6 \mathrm{MeV}$ & 14 & 73.7 \\
\hline 6MeV Photons/VMAT & I & 5.3 \\
\hline Brachytherapy & 0 & \\
\hline Total & 19 & 100 \\
\hline \multicolumn{3}{|c|}{ Dosage (Gy/fraction) } \\
\hline $50 / 20$ & 25 & I5.I \\
\hline $45 / 15$ & 12 & 7.3 \\
\hline $60 / 30$ & 19 & 10.3 \\
\hline $55 / 25$ & 16 & 9.7 \\
\hline $45 / 20$ & 9 & 5.4 \\
\hline Others & 84 & \\
\hline
\end{tabular}

\section{Outcomes}

Cosmetic and functional outcomes were often not explicitly reported. The recurrence rate was considered a proxy measure for efficacy. Figure 5 plots the recurrence rate during follow-up. Figure 6 is an example of definitive RT for lentigo maligna (LM) of the eyelid using SXRT with good cosmetic and functional outcomes 1-year post RT. Figure 7 is an example of definitive RT for BCC of the lower eyelid using SXRT with good cosmetic and functional outcomes six months post RT. Figure 8 shows definitive SXRT treatment of a lip SCC with good cosmetic and functional outcomes three months post RT (Table 5).

Table 5 Recurrence Outcomes $(n=8)$

\begin{tabular}{lll}
\hline Ear Recurrences & Number & Percentage \\
\hline Recurrence Status & & \\
No recurrence & 87 & 94.6 \\
Local recurrence & 3 & 3.2 \\
Regional recurrence & 2 & 2.2 \\
\hline
\end{tabular}


Table Continued...

\begin{tabular}{lll}
\hline Ear Recurrences & Number & Percentage \\
\hline Time to Local Recurrence & & \\
Mean & $\begin{array}{l}26 \text { weeks (6 } \\
\text { months) }\end{array}$ & \\
& $\begin{array}{l}13 \text { weeks (3 } \\
\text { months) }-36 \\
\text { weeks (8 months) }\end{array}$ & \\
Range & \\
Method of Diagnosing Local & \\
Recurrence & \\
Clinical & 0 & 0 \\
Biopsy & 3 & 100 \\
Salvage Therapy Used & & 100 \\
Surgery & 3 & 0 \\
Others & 0 & \\
\hline
\end{tabular}

\begin{tabular}{ll}
\hline Lip Recurrences & \\
\hline Number Percentage \\
\hline
\end{tabular}

\section{Recurrence Status}

$\begin{array}{lcc}\text { No recurrence } & 32 & 88.9 \\ \text { Local recurrence } & 3 & 8.3 \\ \text { Regional recurrence } & \mathrm{I} & 2.8\end{array}$

Time to Local Recurrence

$\begin{array}{ll}\text { Mean } & 117 \text { weeks ( } 26 \text { months) } \\ \text { Range } & \begin{array}{l}9 \text { weeks ( } 2 \text { months) }-274 \text { weeks (6I } \\ \text { months) }\end{array}\end{array}$

\section{Method of Diagnosing Local Recurrence}

$\begin{array}{lll}\text { Clinical } & \text { I } & 25 \\ \text { Biopsy } & 2 & 75\end{array}$

Salvage Therapy Used

$\begin{array}{lll}\text { Surgery } & 2 & 50 \\ \text { Radiotherapy } & 2 & 50 \\ \text { Others } & 0 & 0\end{array}$

Eyelid

Recurrences

\begin{tabular}{lll}
\hline & Number & Percentage \\
\hline Recurrence Status & & \\
No recurrence & 14 & 74 \\
Local recurrence & 2 & 11 \\
Regional recurrence & 3 & 15 \\
\hline
\end{tabular}

Table Continued

\begin{tabular}{|c|c|c|}
\hline $\begin{array}{l}\text { Eyelid } \\
\text { Recur }\end{array}$ & & \\
\hline & Number & Percentage \\
\hline Time & rence & \\
\hline Mean & $\begin{array}{l}43 \text { weeks } \\
\text { months) }\end{array}$ & \\
\hline Range & $\begin{array}{l}21 \text { weeks } \\
\text { (14 month }\end{array}$ & s) -61 weeks \\
\hline
\end{tabular}

Method of Diagnosing Local Recurrence

$\begin{array}{lll}\text { Clinical } & \text { I } & 50 \\ \text { Biopsy } & \text { I } & 50\end{array}$

Salvage Therapy Used

$\begin{array}{lll}\text { Surgery } & 0 & 0\end{array}$

Radiotherapy $\quad 0 \quad 0$

Observation $\quad$ I $\quad 50$

Immunomodulated 0

Hedgehog pathway inhibitor (HPI)*

50

*Vismenogib (HPI) was administered for BCC of the eyelid which had invaded bone. It was started in September 2017, five months following radiotherapy completion.

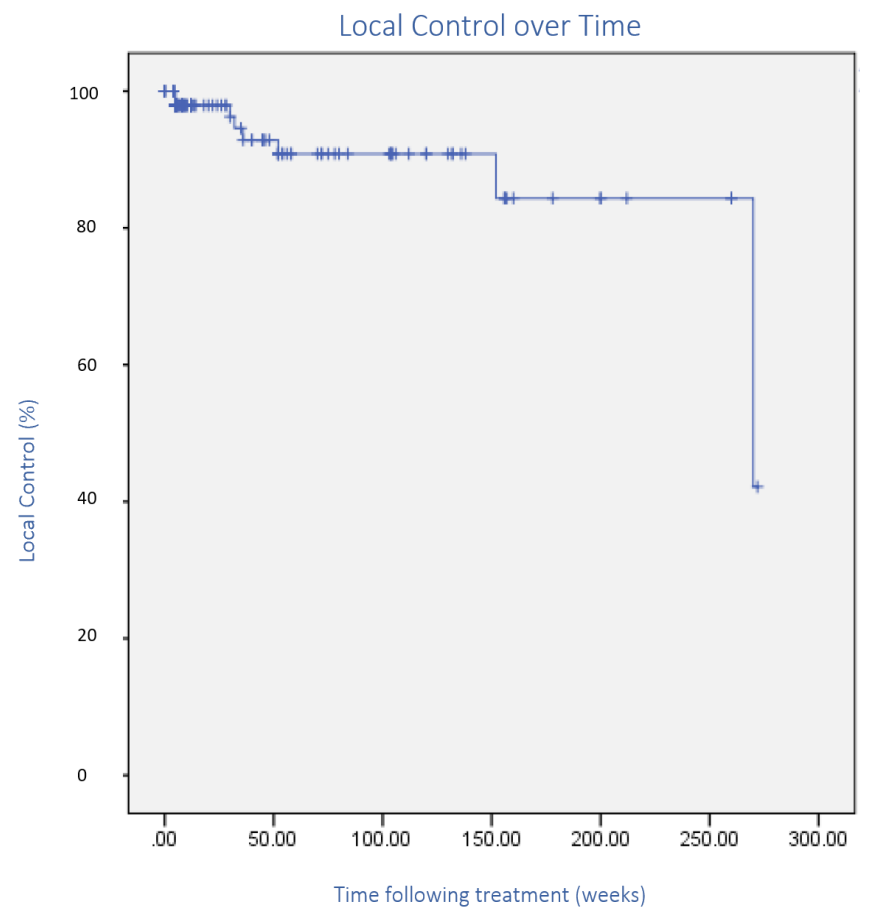

Figure $\mathbf{5}$ Kaplan-Meier plot of recurrence over time. The large drop is due to a recurrence $6 \mathrm{I}$ months following treatment. 
Figure 6 Definitive RT for lentigo maligna (LM) of the eyelid using SXRT.

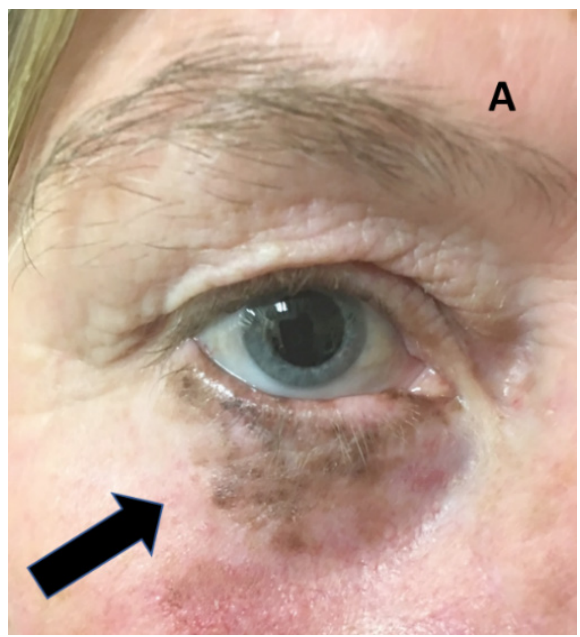

Figure 6A Biopsy proven LM at presentation.

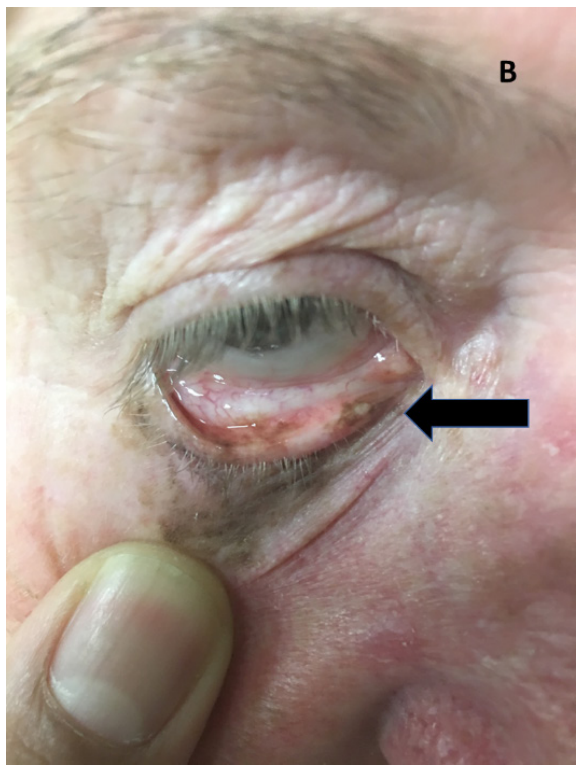

Figure 6B LM spills over onto palpebral conjunctiva.

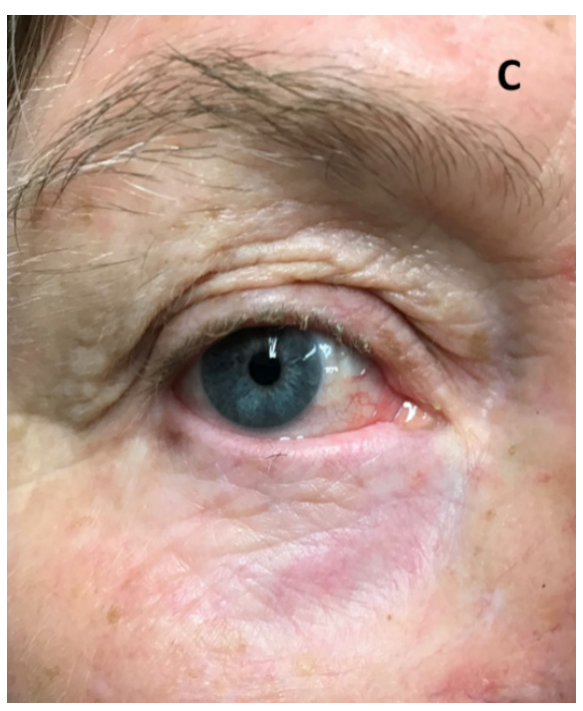

Figure 6C Complete in-field response with optimal functional and cosmetic outcome I-year post radiotherapy.

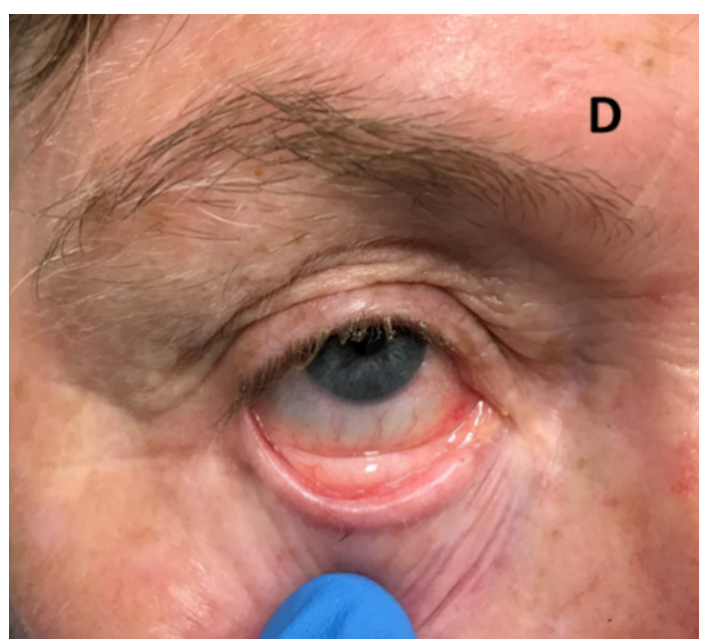

Figure 6D Complete resolution of conjunctival LM I-year post radiotherapy.

Figure 7 Definitive RT for BCC of the eyelid using SXRT.

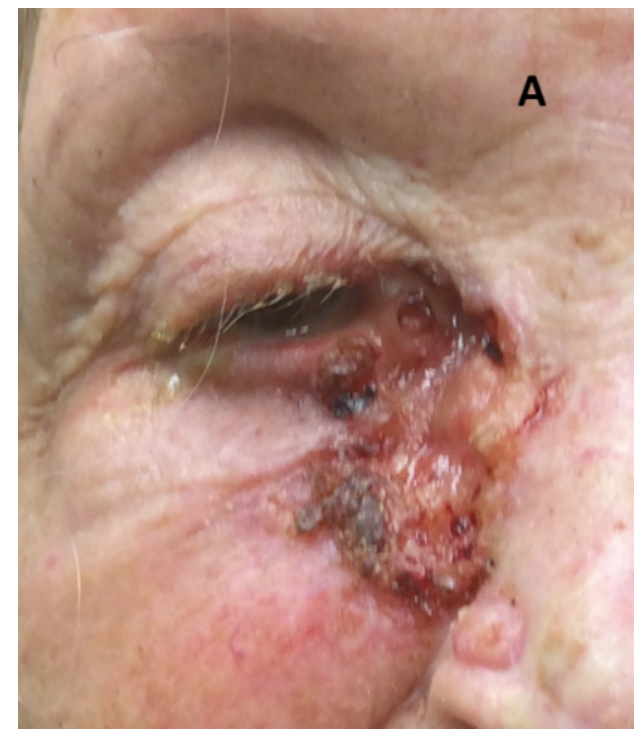

Figure 7A Biopsy proven BCC of medial lower eyelid.

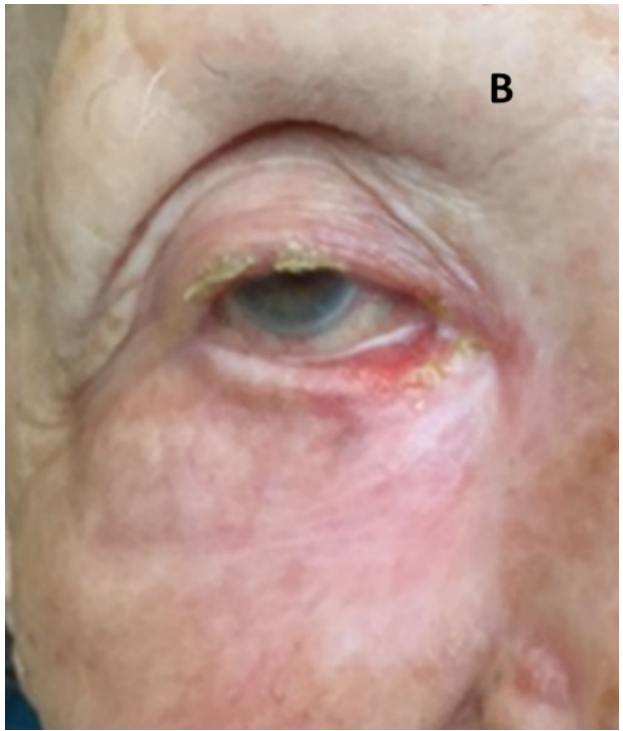

Figure 7B Complete resolution six months after definitive SXRT.

Citation: Tanous A, Tighe D, Bartley J, et al. Lesion-based radiotherapy of the ears, lips and eyelids for skin cancer. Int J Radiol Radiat Ther. 202 I;8(I):32-42. DOI: I0.15406/ijrrt.202I.08.00292 
Figure 8 Definitive treatment of a SCC on the lip with SXRT.

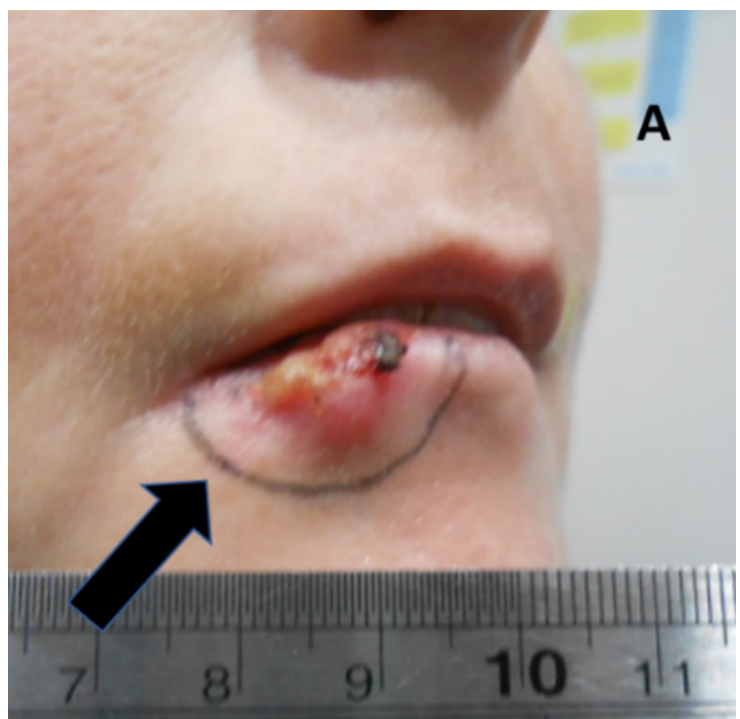

Figure 8A T2NO cSCC lower left lip on presentation. Arrow shows SXRT area.

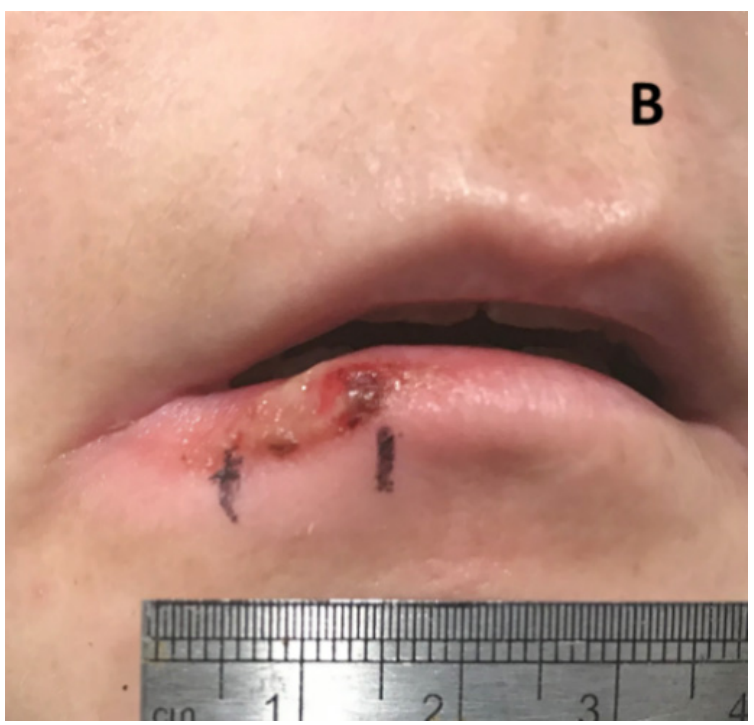

Figure 8B One-month post SXRT and the tumour still persists.

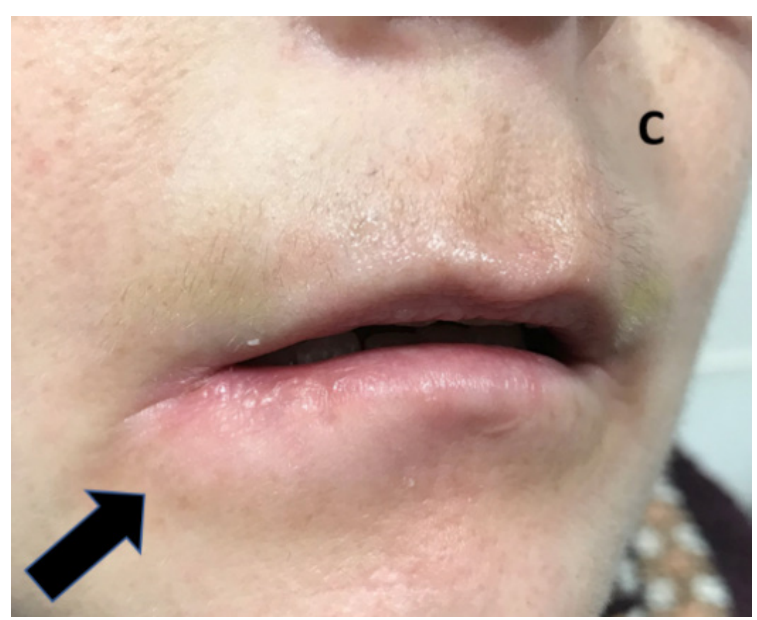

Figure $\mathbf{8 C}$ After three months all the tumour has resolved.

\section{Local recurrence}

Eight local recurrences were identified following RT. The mean time to recurrence was 17 months (range: 3-61 months). Of the eight lesions with local recurrence, five were previously recurrent lesions. Treatment was definitive for six of the recurrences. The pinna had a recurrence rate of $3.2 \%(3 / 92)$, the lip had a recurrence rate of $9.3 \%$ $(3 / 32)$ and the eyelid had a recurrence rate of $10 \%(2 / 21)$. Five BCCs and three cSCCs recurred.

Positive risk factors for recurrence were identified. One 55-year old male who was treated for a BCC on the eyelid was found to have bone involvement which caused persistent and recurrent disease. Another patient had two treatment courses to the lip, but both resulted in recurrence. This patient was 47-years old at his first treatment and 48 at the time of the second treatment event. He had extra-tumoral invasion with additional perineural invasion and muscle infiltration. One patient had a cyst develop on the posterior pinna following treatment. Initially, it was believed to be an atypical presentation of recurrent $\mathrm{BCC}$; however, upon biopsy, the lesion was found to be a trichilemmal carcinoma. This trichilemmal carcinoma was one $\mathrm{cm}$ in diameter with a raised edge of seven $\mathrm{mm}$ (Figure 9). The lesion was completely in-field. Finally, one patient who had undergone treatment to the eyelid for BCC had persistent disease following treatment. This patient had bone involvement due to BCC invasion prior to RT.

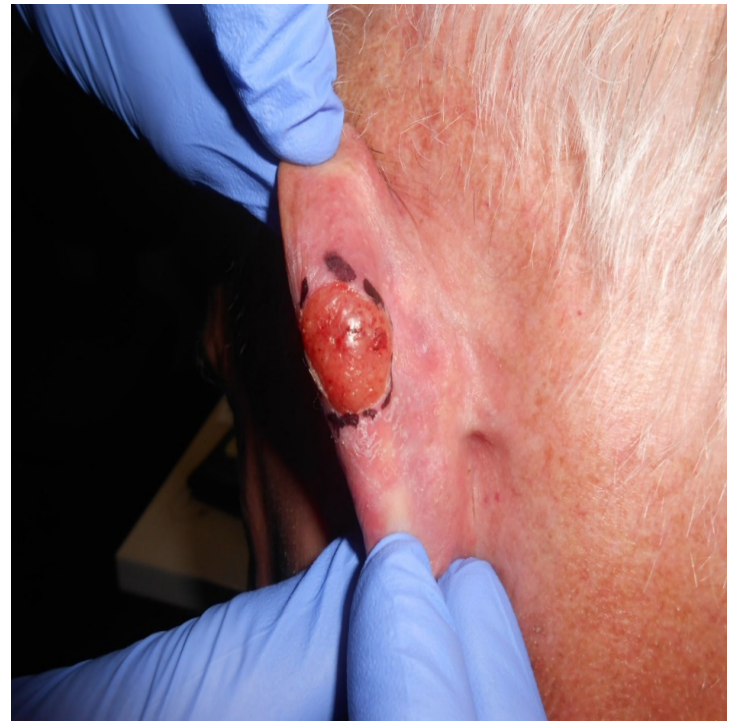

Figure 9 Trichilemmal carcinoma following treatment of an initial BCC. This carcinoma was in-field but only appeared following irradiation to treat the initial BCC lesion.

\section{Adverse reactions}

Twenty-six cases of late side effects following treatment were documented (Table 6). Four patients reported difficulty hearing following radiotherapy and suffered from excessive wax buildup and marked inflammation of the external auditory meatus. A patient who underwent four surgical procedures, and still displayed persistent recurrences with perineural invasion, had skin breakdown and subsequent tragus lymphoedema. Telangiectasia was noted in three patients (two ears and one lip), and two of these had received hypofractionated treatment (Figure 10).

Figure 11 shows an 85-year old man with ECOG 3 performance status who was treated palliatively for a superficial symptomatic Bowen's disease of the ear centred around the rim of the pinna. At one-month post RT, there was complete symptomatic and clinical 
resolution. The same patient presented several months later with this tender ulcer on the RT field edge. At first, this may appear to be a recurrent or de novo cancer, but it was, in fact, a benign ulcer caused by the patient sleeping on this ear. Note that this is the most protuberant part of the ear and would take all the weight of the head on the pillow. Biopsy or RT would prolong healing. The solution is to take the weight off the ear by changing the sleeping posture or, if this is not possible, to treat the situation in the same manner as a corn on the foot by applying special bandages that remove the weight off the lesion during sleep.

Table 6 Description of late side effects $(n=26)$

\begin{tabular}{lll}
\hline Late Side Effects & & \\
\hline No Late Side Effects & 139 & 84.4 \\
Ear Side Effects & & \\
Alopecia & 4 & 2.4 \\
Excessive wax build-up & 4 & 2.4 \\
Telangiectasia & 2 & 1.2 \\
Tragus lymphoedema & 1 & 0.6 \\
Xerostomia & $\mathrm{I}$ & 0.6 \\
\hline
\end{tabular}

\section{Lip Side Effects}

$\begin{array}{lll}\text { Abnormal cosmesis } & 4 & 2.4 \\ \text { Ulceration } & 3 & 1.8 \\ \text { Excessive salivary production } & \mathrm{I} & 0.6 \\ \text { Telangiectasia } & \mathrm{I} & 0.6 \\ \text { Altered sensation } & \mathrm{I} & 0.6 \\ \text { Eyelid Side Effects } & & \\ \text { Abnormal cosmesis } & 2 & 1.2 \\ \text { Inflammation } & 2 & 1.2 \\ \text { Total } & 165 & 100\end{array}$

Figure 10 Late effects in lips.

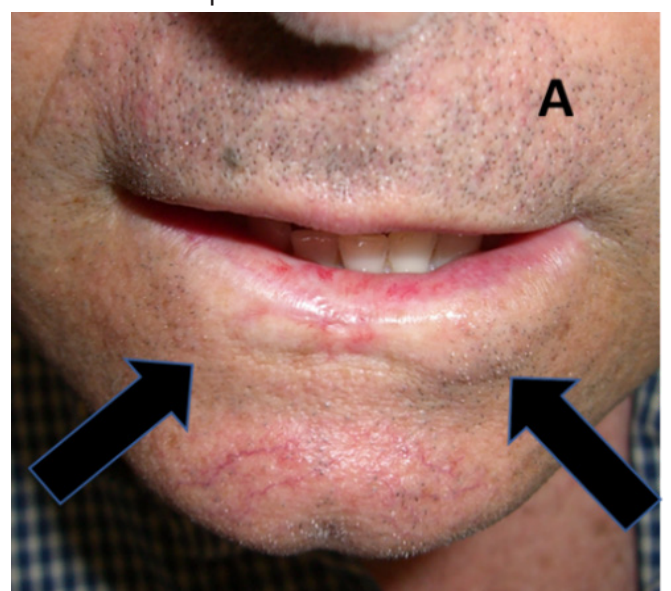

Figure I0A Lip treated with hypofractionated PORT showing tissue loss, hypopigmentation and telangectasia. Arrows show RT field borders.

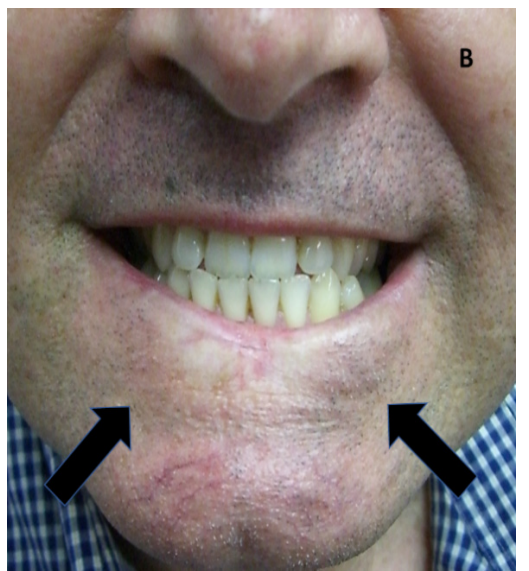

Figure IOB Same patient as in IOA demonstrating a smile following hypofractionation. This technique has resulted in a poorer cosmetic outcome. Arrows show RT field borders.

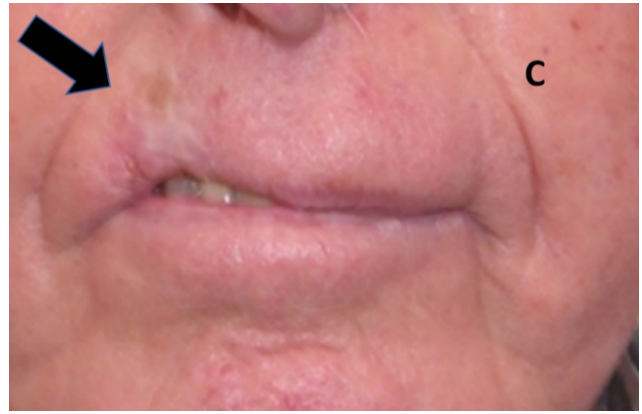

Figure I0C Lip treated with hypofractionated definitive RT showing tissue loss, cicatrisation, hypopigmentation and telangiectasia. Arrow shows RT area.

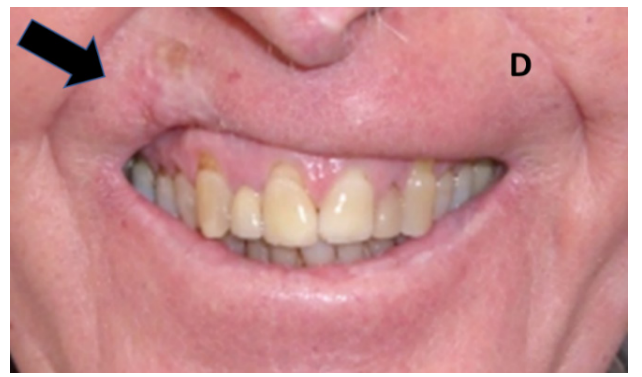

Figure IOD Same patient as in I0C. Definitive hypofractionation has also impacted function causing an asymmetrical smile. Arrow shows RT area.

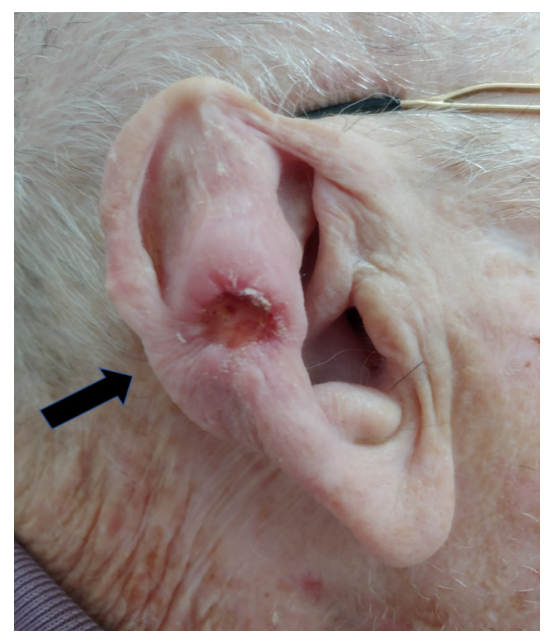

Figure II Photo of right ear from direct lateral showing benign ulcer (black arrow). 


\section{Referral growth}

In years gone by, radiotherapy, at least in Australia, was a component of dermatology practice. With the advent of Mohs surgery and enhanced surgical techniques, the use of radiotherapy waned. Modern radiation therapy with its new techniques is seeing a resurgence in the management of non-melanoma skin cancers but gaining the confidence of referrers takes time. This study highlights the growth of a single clinician radiotherapy skin practice over time (Figure 12), and the ratio of definitive to post-operative referrals (Table 7), showing increased referrer's knowledge of the efficacy of definitive RT alone for skin cancer management.

\section{Growth of Referrals over Time}

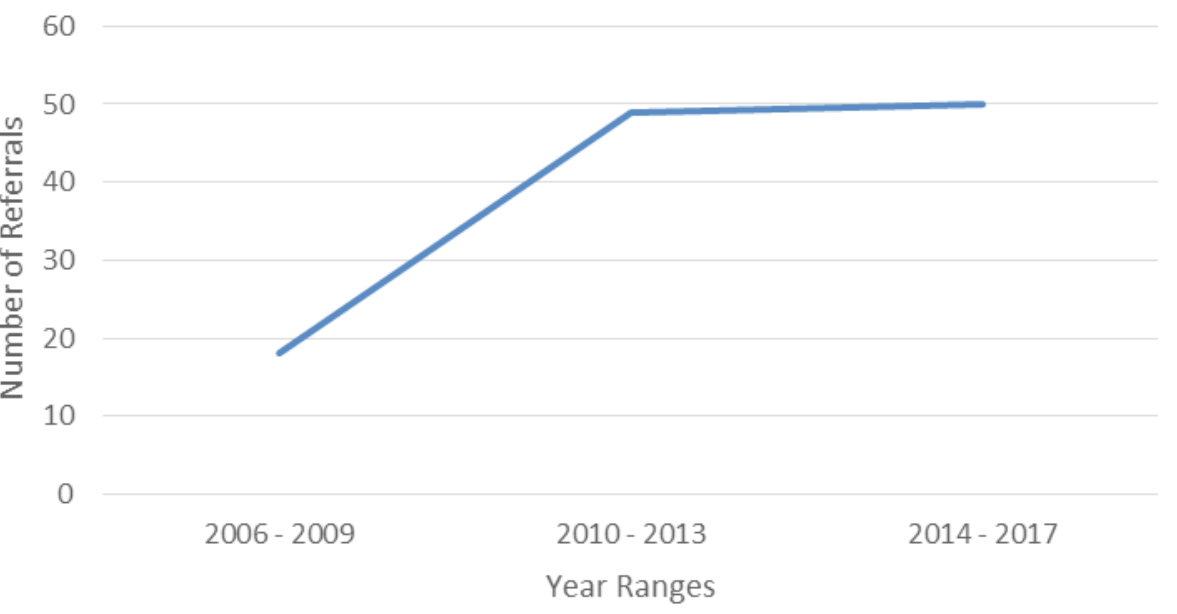

Figure 12 Referral growth over time.

Table 7 Increase in RT referrals for ears only over time. Note that the ratio of definitive to adjuvant intent increases over time as the referrer's knowledge of the efficacy of RT grows

\begin{tabular}{llll}
\hline Year & Definitive Intent & Adjuvant Intent & Ratio of Definitive/Adjuvant Intent \\
\hline $2007-2011$ & 6 & 9 & 0.66 \\
$2012-2016$ & 24 & 18 & 1.33 \\
$2017-$ present & 28 & 8 & 3.5 \\
Total & $\mathbf{5 8}$ & $\mathbf{3 5}$ & \\
\hline
\end{tabular}

\section{Discussion}

This paper is a retrospective audit of a single clinician's treatment of skin cancer of the ear, eyelid, and lip with RT. One hundred and forty-seven patients with 165 lesions were treated over a ten-year period. Definitive RT was given in $108(65.5 \%)$ cases with a median follow-up of 42 weeks. The recurrence rate was $8 / 165$ or $5 \%$. Six cases were successfully salvaged with either surgery (5) and / or RT (2). One was treated with vismenogib, another was treated with palliative intent. Late side effects were seen in 26 patients. This study also identified the growth in referrals for a tertiary service over time.

A difference in incidence according to gender was found as $79.8 \%$ of the treated patients were male. Males have a higher incidence of keratinocytic cancer and morbidity compared to females. ${ }^{1,7-9}$ A possible explanation for this is that long hair is more common in females than in males which may reduce the cumulative life-time exposure of UV light, particularly to the ear. Cook et al. found that males have worse skin cancer survival compared with females from adolescence right through all age groups. ${ }^{8,10,11}$ Basal cell carcinoma and squamous cell carcinoma have been shown to have higher rates of morbidity and mortality in men. ${ }^{12-14}$

This analysis showed an almost similar incidence of BCC to cSCC. The ear is a high-risk site and the current literature provides diverse results. Silva et al. found a ratio of 1.65:1 of BCC vs $\mathrm{cSCC}^{15}$ in a collection of 313 lesions, whilst Duffy et al. reported a ratio of 1:3 in 142 lesions. ${ }^{16}$ In our retrospective data, the ratio between $\mathrm{cSCC}$ and BCC was 1:1 for the ears which is atypical compared to other sites. For eyelids, we found a ratio between BCC versus CSCC of 1.44: 1 . However, the literature suggests that $\mathrm{cSCC}$ is more common on the lower lip. ${ }^{17,18}$ Our data contradicts this and suggests that the trend of increased $\mathrm{BCC}$ vs cSCC remains in the lips $(1.62: 1)$; however, this may be due to referrers preferentially sending cSCC for surgery.

We found a local recurrence rate of five percent with eight infield recurrences between 3-61 months post treatment. Of the eight recurrences, six occurred when treated with definitive intent. This suggests that PORT decreases the risk of recurrence following surgery for higher risk lesions. The ear had the lowest recurrence rate, but this may be attributed to the volume of patients treated for skin cancer of the ear compared to the eyelids and lips.

According to Thomson et $\mathrm{al},{ }^{19}$ two prospective trials have considered RT for BCC and, of these, only one compared RT to surgery for the treatment of BCC. This was published by Avril et al. in 1997 and observed the difference in local recurrence and cosmetic outcome between surgery and RT of the face. The local recurrence rate at four years following surgical treatment was $0.7 \%$ compared to 
RT at 7.5\%. In Avril et al.'s prospective trial, 11, 70 and 22 of the 374 patients treated with RT or surgery underwent treatment for BCC of the ear, eyelids and superior only lip, respectively. ${ }^{20}$

Our data provides some evidence that RT is an acceptable treatment method for high-risk sites. Our evidence shows a local recurrence rate that is comparative to Arvil et al. (5\% vs $7.5 \%$, respectively) when a more focussed treatment site is targeted. Moreover, in the RT arm in Avril et al.'s study, most patients were treated with interstitial brachytherapy, whereas in our study, external beam RT was the predominant modality with brachytherapy was reserved only for the treatment of benign keloids. More current and focussed data on the use of RT for skin cancer is needed in various settings.

Ashby et al. conducted a retrospective Australian study of 1154 cases of keratinocytic cancer treated at the Peter MacCallum Cancer Institute (Melbourne, Victoria). ${ }^{3}$ Univariate and multivariate analysis found that the ear and the eyelid had the highest recurrence rate of any anatomical site.

Figure 12 and Table 7 give important data about changing referral patterns for RT. Figure 12 shows that referrals for RT increased over time as referrers gained confidence. More importantly, Table 7 shows that the ratio of definitive to post-operative referrals grew over time, highlighting the gain in referrer confidence and belief that definitive RT alone could deliver excellent oncological outcomes without the tissue sacrifice associated with surgery.

This audit has several limitations. It is retrospective, the sample size is small, and data comes from a single clinician. Patient followup also varied. Patients who underwent adjuvant therapy received four weeks of follow-up before returning to their referrer's practice for ongoing surveillance, whereas definitive patients generally had more long-term follow-up with the treating radiation oncologist. Incomplete recording of tumour factors limited the scope of the study. Histological reporting also varied and was often incomplete.

This data, however, displays the role of RT in treating lesions in high-risk sites with acceptable outcomes. It also shows the growth of a skin specific radiotherapy clinic as referrer confidence increased. ${ }^{6}$ With more modern treatment options, optimisation of data collection, and accurate representation of histological data, RT has become an important treatment method for keratinocytic cancer. Given the the absence of prospective research and the importance of tailoring treatment to high-risk sites, this retrospective data may inform future prospective trials comparing RT with other treatment modalities.

\section{Conclusion}

This retrospective, single-clinician audit of 149 patients with 165 lesions on the ear, eyelids and lips treated with radiotherapy supports the use of radiotherapy to treat keratinocytic cancer, even in the definitive setting. This study also highlights the growth of a single clinician radiotherapy skin practice over time and the ratio of definitive to post-operative referrals, showing increased confidence in definitive RT alone for skin cancer management. Future prospective research is required to obtain higher quality evidence to further guide treatment in these high-risk sites.

\section{Conflicts of interest}

The authors have no conflicts of interest to declare.

\section{Acknowledgments}

The authors wish to thank Aileen Eiszele of A\&L Medical Communications for editing the manuscript and for overseeing the journal submission process. Thanks also go to Duncan Mccrae of GenesisCare for posing as a model for Figure 2.

\section{References}

1. Australian Institute of Health and Welfare. Skin cancer in Australia. Department of Health, Canberra; 2016.

2. Meaike JD, Dickey RM, Killion E, et al. Facial skin cancer reconstruction. Seminars in Plastic Surgery. 2016;30:108-121.

3. Ashby MA, Smith J, Ainslie J, et al. Treatment of nonmelanoma skin cancer at a large Australian center. Cancer. 1989;63:1863-1871.

4. Drake RL, Vogl W, Mitchell A. Gray’s basic anatomy. 2nd edn. 2017.

5. National Cancer Institute. Common terminology criteria for adverse events (CTCAE), Version 5, 2010.

6. Damian DL, Matthews YJ, Phan TA et al. An action spectrum for ultraviolet radiation-induced immunosuppression in humans. $\mathrm{Br}$ JDermatol. 2011;164:657-659.

7. Gamba CS, Clarke CA, Keegan TH et al. Melanoma survival disadvantage in young, non-Hispanic white males compared with females. JAMA Dermatol. 2013;149:912-920.

8. Sideris E, Thomas SJ. Patients' sun practices, perceptions of skin cancer and their risk of skin cancer in rural Australia. Health PromotJ Austr. 2020;31;84-92.

9. Liu-Smith F,Farhat AM, Arce A, et al. Sex differences in the association of cutaneous melanoma incidence rates and geographic ultraviolet light exposure. J Am Acad Dermatol. 2017;76:499-505.

10. Cook MB, McGlynn KA, Devesa SS, et al. Sex disparities in cancer mortality and survival. Cancer Epidemiol Biomark Prev. 2011;20:16291637.

11. Perera E, Gnaneswaran N, Staines C, et al. Incidence and prevalence of non-melanoma skin cancer in Australia: A systematic review. Aust $J$ Dermatol. 2015;56:258-267.

12. Turner SJ, Morgan GJ, Palme CE, et al. Metastatic cutaneous squamous cell carcinoma of the external ear: a high-risk cutaneous subsite. $J$ Laryngol Otol. 2010;124:26-31.

13. Kang KW, Lee DL, Shin HK, et al. A retrospective clinical view of basal cell carcinoma and squamous cell carcinoma in the head and neck region: a single institution's experience of 247 cases over 19 years. Arch Carniofac Surg. 2016;17:56-62.

14. Silva JJ, Tsang RW, Panzarella T, et al. Results of radiotherapy for epithelial skin cancer of the pinna: The Princess Margaret Hospital experience, 1982-1993. Int J RadiatOncolBiol Phys. 2000;47:451-459.

15. Duffy KL, McKenna JK, Hadley ML, et al. Nonmelanoma skin cancers of the ear: correlation between subanatomic location and post-mohs micrographic surgery defect size. Dermatol Surg. 2009;35:30-33.

16. Cerezo L, Liu FF, Tsang R, et al. Squamous cell carcinoma of the lip: analysis of the Princess Margaret Hospital experience. RadiotherOncol. $1993 ; 28: 142-147$

17. dos Santos LR, Cernea CR, Kowalski LP, et al. Squamous-cell carcinoma of the lower lip: a retrospective study of 58 patients. Sao Paulo Med Med J. 1996;114:1117-1126.

18. Thomson J, Hogan S, Leonardi-Bee J, et al. Interventions for basal cell carcinoma of the skin. Cochrane Database Syst Rev.2020;(1):CD003412.

19. Avril MF, Auperin A, Margulis A, et al. Basal cell carcinoma of the face: surgery or radiotherapy? Results of a randomized study. Br J Cancer. 1997;76(1):100-106.

20. Fogarty GB, Christie DRH, Wong B, et al. Experiences in growing a skin radiation therapy practice. Int J RadiolRadiatTher. 2020;7(6):168-182. 